\title{
An loT measurement solution for continuous indoor environmental quality monitoring for buildings renovation
}

\author{
Serena Serroni ${ }^{1}$, Marco Arnesano ${ }^{2}$, Luca Violini ${ }^{1}$, Gian Marco Revel ${ }^{1}$ \\ ${ }^{1}$ Università Politecnica delle Marche, Department of Industrial Engineering and Mathematical Science, Via delle Brecce Bianche, \\ 60131 Ancona (AN), Italy \\ 2 Università eCampus, Via Isimbardi 10, 22060 Novedrate (CO), Italy
}

\begin{abstract}
The measurement of Indoor Environmental Quality (IEQ) requires the acquisition of multiple quantities regarding thermal comfort and indoor air quality. The IEQ monitoring is essential to investigate the building's performance, especially when renovation is needed to improve energy efficiency and occupants' well-being. Thus, IEQ data should be acquired for long periods inside occupied buildings, but traditional measurement solutions could not be adequate. This paper presents the development and application of a non-intrusive and scalable IoT sensing solution for continuous IEQ measurement in occupied buildings during the renovation process. The solution is composed of an IR scanner for mean radiant temperature measurement and a desk node with environmental sensors (air temperature, relative humidity, $\mathrm{CO} 2, \mathrm{PMs})$. The integration with a BIM-based renovation approach was developed to automatically retrieve building's data required for sensor configuration and KPIs calculation. The system was installed in a nursery located in Poland to support the renovation process. IEQ performance measured before the intervention revealed issues related to radiant temperature and air quality. Using measured data, interventions were realized to improve the envelope insulation and the occupant's behaviour. Results from postrenovation measurements showed the IEQ improvement achieved, demonstrating the impact of the sensing solution.
\end{abstract}

\section{Section: RESEARCH PAPER}

Keywords: Thermal comfort; indoor air quality; IEQ; measurements; IoT; building renovation

Citation: Serena Serroni, Marco Arnesano, Luca Violini, Gian Marco Revel, An IoT measurement solution for continuous Indoor environmental quality monitoring for buildings renovation, Acta IMEKO, vol. 10, no. 4, article 35, December 2021, identifier: IMEKO-ACTA-10 (2021)-04-35

Section Editor: Carlo Carobbi, University of Florence, Gian Marco Revel, Università Politecnica delle Marche and Nicola Giaquinto, Politecnico di Bari, Italy Received October 10, 2021; In final form December 10, 2021; Published December 2021

Copyright: This is an open-access article distributed under the terms of the Creative Commons Attribution 3.0 License, which permits unrestricted use, distribution, and reproduction in any medium, provided the original author and source are credited.

Funding: This work was supported by P2Endure, European Project Horizon 2020.

Corresponding author: Serena Serroni, e-mail: s.serroni@pm.univpm.it

\section{INTRODUCTION}

The measurement of Indoor Environmental Quality (IEQ) requires the acquisition of multiple quantities regarding thermal comfort and indoor air quality. Therefore, accurate monitoring and control of those environmental conditions can be useful for preventing the spread of COVID-19. However, about $75 \%$ of European buildings stock was built before 1990, before any EU building regulation [1] and with a climate context that has changed through the last decade [2]. Thus, most of occupied buildings are not able to keep the required comfort conditions because of the poor performance of the envelope and heating/cooling systems [3].

The importance of Indoor Environmental Quality (IEQ) is a well-known and largely discussed theme because of its impact on human comfort, well-being, productivity, learning capability and health [4]. IEQ derives from the combination of different factors influencing the human comfort sensation: Thermo hygrometry, acoustic, illumination and concentration of pollutant components [5]. All those aspects should be considered at the same level of importance considering that humans spend roughly $90 \%$ of their time indoors, especially after COVID-19 spread. In addition, a recent study [6] demonstrated that indoor environmental factors, such as temperature, humidity, ventilation, and filtering systems could have a significant influence on the infection. Several studies showed a correlation between the concentration of air pollutants, especially particular matter 2.5 (PM2.5) and particular matter 10 (PM10), and COVID-19 virus transmission [7].

For this reason, current buildings' renovation approaches and trends are including the IEQ in the renovation assessment with 
an increased role of importance [8]. Recently, the standard EN 16798 [9] has been released, in substitution of the EN 15251, which provides a framework for the building performance assessment concerning the indoor environment. EN 16798 provides methodologies for the calculation of IEQ metrics for buildings' classification, based on environmental measurements [10]. In that standard, thermal comfort is assessed according to well-known predictive and adaptive approaches, as defined in ISO 7730 [11] and ASHRAE 55. However, the same level of detail is not given to indoor air quality, visual comfort, and acoustic comfort. In their critical review [12], Khovalyg et al remarked that ISO and EN standards should include more requirements on PM. Similarly, another critical investigation on IEQ data collection and assessment criteria presented in [13] revealed that sensor technology and data analysis are mainly applied to thermal comfort, while other IEQ's domains have not been addressed with the same effort. For this reason, the assessment of IEQ domains, unconsidered by standards, has been conceptualized within recent research activities [14] also with a holistic approach that groups domains together [15].

Even if the importance of IEQ has been largely demonstrated, actual measurement tools are not adequate because of the need of measuring several environmental quantities with a high temporal and spatial resolution. Traditional spot measurements tools are bulky and require a strong human effort for data processing and analysis. So, they can't be used for responsive strategies to improve IEQ by implementing retrofit actions (envelope insulation, mechanical ventilation) or triggering occupants' behaviours (windows opening, thermostat regulation). Recently, the use of sensors integrated into building management systems (BMS) has also been investigated for IEQ monitoring [16].However, those systems generally make use of wall-mounted sensors providing environmental quantities measured away from the real location of the occupants. Therefore, data could be representative of a small part of the building. Optimization of the sensing location could be performed for some quantities, such as air temperature [17], but the same optimization could be difficult for quantities that present relevant deviations in the same room, as the case of mean radiant temperature. The mean radiant temperature is not homogeneous in the room and depends on the indoor walls' temperature [18]. In the proximity of a glazed surface or of a poorly insulated wall, the mean radiant temperature presents higher variations with respect to the inner side of the same room [19]. ISO 7726 [20] provides two methods for measuring the mean radiant temperature: i) the globe thermometer that is basically a temperature sensor located at the centre of a matt black sphere; ii) the angle factors approach, based on the walls' temperature measurements. The globe thermometer is widely used for spot measurements. It is intrusive, provides data only related to the position where it is located and one measurement point could not be enough to determine the real room's comfort. Thus, is not the preferable solution for continuous IEQ measurements. The second approach, based on the angle factors, could provide a higher resolution once that the problem of measuring the walls' thermal maps is solved. To this scope, Revel et al. [21] developed an infrared (IR) scanner that provides continuous measurement of indoor walls temperatures for mean radiant temperature calculation according to ISO 7726 . The proposed sensor turned out to provide a measurement accuracy of $\pm 0.4{ }^{\circ} \mathrm{C}$ with respect to traditional microclimate stations [22].

The IR scanner was integrated with a desk node that measures the air temperature and relative humidity to build the solution named Comfort Eye, a sensor for multipoint thermal comfort measurement in indoor environments. That system provides a solution to the problem of measuring real-time thermal comfort with an increased spatial resolution. The impact on heating control efficiency was demonstrated by experimental testing presented in [23].

This paper presents the new development of the Comfort Eye for the continuous monitoring of IEQ for buildings renovation. Indoor air quality (IAQ) sensors have been integrated into the desk node to provide measurements of $\mathrm{CO}_{2}$ and PMs. Moreover, a LED lighting system has been embedded in the desk node to provide occupants with feedback about the actual status of the indoor air quality.

An Internet of Things (Io'T) architecture has been developed to allow remote configuration and data exchange. Interoperability with BIM (Building Information Model) has been developed to automatically retrieve building's data (e.g., floor area, geometry, material emissivity, occupancy, etc.), needed for sensors configuration, metrics calculation and performance assessment.

The most important differences compared to the previous version presented in the paper [21] are: new IR sensor; a new sensor for IAQ, in particular, new $\mathrm{CO}_{2}$ and PMs sensor; the last version presents an updated architecture, it is Plug\&Play and IoT; Integration with the BIM to configure automatically the sensors and KPIs calculation.

The advanced version of the Comfort Eye was developed within the framework of the European project, P2Endure. P2Endure aims at including the IEQ in the renovation process, in all the stages of the $4 \mathrm{M}$ (Mapping, Modelling, Making, Monitoring) process. This means that a protocol for the IEQ monitoring, and assessment has been developed, allowing: the accurate evaluation of IEQ performance of the building as it is to feed the design stage with the suggestions to achieve the optimal IEQ level after the intervention, and the post-renovation monitoring to verify the achievement of IEQ compliance according to issues revealed with the mapping. The Comfort Eye was applied for the continuous IEQ monitoring of a building located in Gdynia (Poland) before and after renovation works.

The aspects of IEQ that are taken into consideration, using the Comfort Eye, and exploiting all its potential, are thermal comfort and IAQ $\left(\mathrm{CO}_{2}\right.$ and $\left.\mathrm{PM}\right)$

Results from the field application are reported. This paper demonstrates the applicability and advantages of the developed system with the application to a real case study. A pre and post renovation analysis was performed to validate the developed monitoring methodology. The monitoring allowed to quantify, in the pre and post renovation phase, the thermal performances of the building, to identify the main causes of discomfort and to assess the IAQ.

The specific goals of the paper are:

- To present the measurement system, sensors specification, IoT architecture and integration with the BIM;

- To propose an IEQ monitoring and assessment protocol that extends the EN 16798 approach to include PMs;

- Demonstrate the applicability and advantages of the proposed measurement device with the application to a real case study. 
Table 1. Specifications of sensors used by the desk node of the Comfort Eye.

\begin{tabular}{lccc}
\hline $\begin{array}{c}\text { Sensirion } \\
\text { SCD30/SPS30 }\end{array}$ & Range & Accuracy & Repeatability \\
\hline $\mathrm{CO}_{2}$ in $\mathrm{ppm}$ & $0-40000$ & $\pm(30 \mathrm{ppm}+3 \% \mathrm{MV})$ & $\pm 10 \mathrm{ppm}$ \\
$R H$ in $\% \mathrm{RH}$ & $0-100$ & \pm 3 & \pm 0.1 \\
$T_{\mathrm{a}}$ in ${ }^{\circ} \mathrm{C}$ & $-40-+70$ & $\pm\left[0.4+0.023\left(T_{\left.\left.{ }^{\circ} \mathrm{C}-25^{\circ} \mathrm{C}\right)\right]}\right.\right.$ & \pm 0.1 \\
$\mathrm{PM} 2.5 \mathrm{in} \mu \mathrm{g} / \mathrm{m}^{3}$ & $0-1000$ & \pm 10 & $/$ \\
$\mathrm{PM} 10$ in $\mu \mathrm{g} / \mathrm{m}^{3}$ & $0-1000$ & \pm 10 & $/$ \\
\hline
\end{tabular}

\section{MATERIALS AND METHODS}

\subsection{The measurement technology}

The Comfort Eye is an IoT sensor composed of two nodes, the ceiling node with the IR sensor to measure walls thermal maps and mean radiant temperature, and the desk node with sensors to measure: air temperature, relative humidity, $\mathrm{CO}_{2}$ and PMs. It can provide the whole thermal dynamic behaviour of the wall for continuous and real-time thermal monitoring of buildings. Being a prototype, the Comfort Eye has a production cost of less than $100 €$ for each node.

This work aims to explore and deepen the functionality of Comfort Eye that allows the measurement of data necessary for the IEQ.

\subsubsection{Comfort Eye-Ceiling Node}

The ceiling node is the innovation of the Comfort Eye. It is a $3 \mathrm{D}$ thermal scanner and to measure temperature maps of all the room's indoor surfaces there are 2-axes rotating IR sensor (Figure 1). It is installed on the ceiling of the room, and it is composed of a $16 \times 4$ thermopile array, meaning that each acquired frame is a map of 64 temperatures. With a horizontal field of view (HFOV) of $60^{\circ}$ and a vertical field of view (VFOV) of $16^{\circ}$, an area of $1.15 \times 0.56 \mathrm{~m}^{2}$ is scanned with one frame on a wall at one meter of distance from the sensor.

The tilt movement with $0^{\circ}-180^{\circ}$ provides the full vertical scanning of the wall with the possibility to measure the floor and ceiling temperatures. To provide the scan of all the surfaces a continuous $360^{\circ}$ pan movement is available. The device entails a custom mainboard with a microcontroller, programmed with dedicated firmware, to perform the automatic scanning of all the room's surfaces by controlling the pan and tilt servos. The $12 \mathrm{C}$ communication protocol is used to acquire data are from the IR sensor. The IR scanner required cabling for a $12 \mathrm{~V}$ power supply while the communication is performed with a Wi-Fi module that is integrated into the mainboard. The scanning process produces one thermal map for each wall that is the result of the concatenation of multiple acquisitions. Given the sensor's FOV, the installation point and the room geometry, the reconstruction of the wall thermal map is performed to remove the overlapping
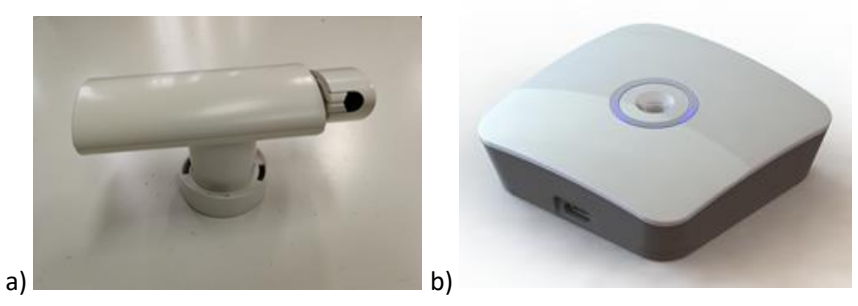

Figure 1. Comfort Eye: a) Ceiling node and b) Desk node. pixels derived from the vertical concatenation and to remove pixels related to the neighbour walls.

Concerning the surface emissivity, correction of the IR raw measurement is implemented [24]. The complete procedure for maps correction is detailed in [21].

The IR data are acquired in continuous, processed and stored in a database in real-time. Corrected thermal maps are then used for two-fold scope: measurement of the mean radiant temperature for thermal comfort evaluation and measurement of building's envelope thermal performance [21]. Mean radiant temperature is measured for several locations (e.g. near and far from the window) with the angle factors method, as presented in ISO 7726 [20].

\subsubsection{Comfort Eye- Desk Node}

A desk node is used to acquire environmental quantities for thermal comfort and indoor air quality (IAQ) assessment (Figure 1).

An integrated sensor, Sensirion SCD30, allows the single point measurement of the air temperature $(\mathrm{Ta})$, relative humidity $(\mathrm{RH})$, and $\mathrm{CO}_{2}$. The desk node also integrates a PM sensor, Sensirion SPS30 (Table 1). Sensors' data are acquired via the I2C interface and a Wi-Fi module, the same that is used for the ceiling node, provides wireless communication. The data are acquired in continuous, processed and stored in the database in real-time. The desk node is in a position that should be representative of the room's environmental conditions, avoiding exposures to direct solar radiation, air droughts and zones characterized by stagnant air.

The desk node must be installed in a position representative of the environmental conditions, away from heat sources, solar radiation, direct ventilation, or other sources that could disturb the measurements. Installation is done by an experienced technician. If possible, the sensor is fixed, otherwise the occupants are informed to prevent the sensor from being moved or covered.

The air quality measurement system has been proposed for real-time, low-cost, and easy to install air quality monitoring. It provides precise and detailed information about the air quality of the living environment and helps to plan interventions that lead to improve air quality.

In crowded closed environments such as classrooms, offices, or meeting rooms, in the case of limited ventilation, $\mathrm{CO}_{2}$ values of between 5,000 ppm and 6,000 ppm can be reached. To have a good air quality the limit of $1000 \mathrm{ppm}$ of $\mathrm{CO}_{2}$ must not be exceeded [25].

An efficient IAQ monitoring system should detect any change in the air quality, give feedback about the measured values of $\mathrm{CO}_{2}$ to the users, and trigger the necessary mechanisms, if available, such as automatic/ natural ventilation and fresh air, to improve performance and protect health. The desk node communicates to the users in real time the measured values of $\mathrm{CO}_{2}$ simply and intuitively, through different colours of the LEDs, green, yellow, red (green for good air quality and red for bad air quality). The value of $\mathrm{CO}_{2}<700 \mathrm{ppm}$ is represented by green LEDs. In this case, the $\mathrm{CO}_{2}$ values are acceptable, there is good air quality, it is not necessary to ventilate the environment. The value of $\mathrm{CO}_{2}$ between $700 \mathrm{ppm}$ and $1000 \mathrm{ppm}$ is represented by yellow LEDs. The $\mathrm{CO}_{2}$ values are very close to the limit value (1000 ppm) and it is recommended to ventilate the environment. The value of $\mathrm{CO}_{2}>1000 \mathrm{pm}$ is represented by red LEDs. The value of $\mathrm{CO}_{2}$ has exceeded the limit value and it is necessary to ventilate the environment [26]. 


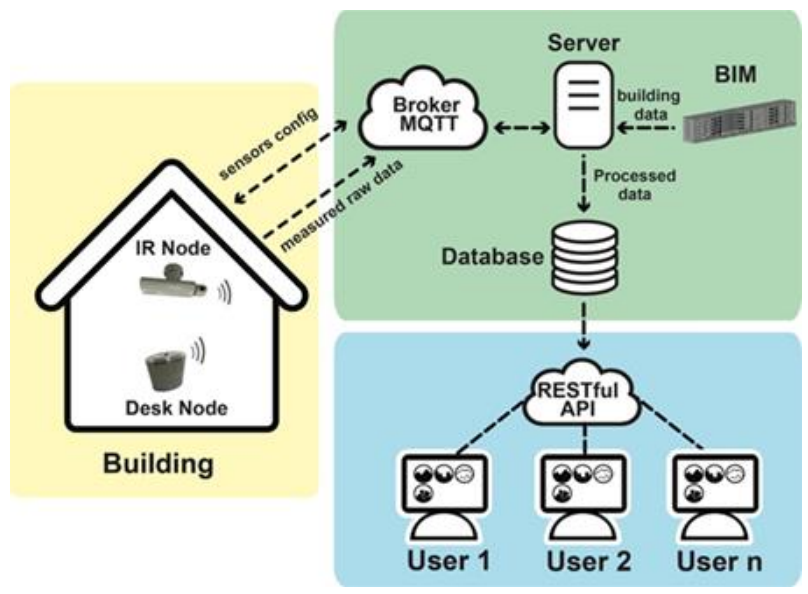

Figure 2. General Architecture of Comfort Eye.

\subsubsection{System Architecture}

Comfort Eye's nodes can be connected through the local WiFi network to the remote server where data are sent, processed, and then stored in a MySQL database. Figure 2 shows general the architecture.

The communication module used is the PyCOM W01. It is an efficient module, which reduces power consumption as implements Bluetooth Low Energy and supports Message Queuing Telemetry Transport (MQTT) protocol that allows only byte communication between the client and server, so to get a light communication, suitable also for low Wi-Fi signal conditions. To integrate the PyCOM W01 a custom Printed Circuit Board (PCB) was developed.

The MQTT communication protocol is used and each node is programmed to be a publisher and subscriber as shown in Figure 2.

The first step, to start the monitoring, is the device configuration with a dedicated mobile application. Configuration data are sent from the mobile device: server information, local Wi-Fi credentials, and room tag. Once the connection is established, the desk node sends directly raw data to the server where a subscriber function provides to processing and storing actions. For the ceiling node, the scanning procedure is configured using geometrical data of the room. Geometry is retrieved from the BIM, as explained in the next subsection. Once the tilt and pan angles have been defined and published to the sensors, the data acquisition starts. The raw data are sent to the server and processed by a subscriber.

The processed data are stored on a MySQL database. The whole monitoring process takes place continuously and in realtime.

The data stored in the database are then available for being consumed. A dashboard for thermal performance assessment and data exploration has been developed. The dashboard is a web-app accessible through any browser. The data processing core is served by a RESTful Application Programming Interface (API) service running on the server and called with standard GET request.

The user can view the measured data via the web-app, obtaining information by consulting the related KPIs, and can have direct feedback on IAQ by observing the LED's color of the desk node installed in the building. The two means of communication are not correlated and independent of each other.

\subsubsection{BIM integration}

Building's data are required to configure the IR scanning process, to perform geometrical maps corrections and to calculate IEQ KPI. To reduce the manual operation, integration with the BIM was developed. The BIM model of a building includes most of the required data, but not those related to the Comfort Eye installation. For this reason, the Comfort Eye BIM object was developed. The object can be imported into the BIM and added to each room where the sensor is installed. Then the IFC file can be exported and processed with a dedicated software based on the ifcopenshell Python library [27]. The software automatically retrieves the data and store them in the MySQL database that is used for the automatic configuration of the Comfort Eye.

The scope of using BIM data is three-fold: i) to correct the IR thermal maps using the room geometry, the sensor position and the wall emissivity; ii) to allow the application of the angle factors method for the mean radiant temperature measurement according to ISO7726 [20]; iii) to calculate IEQ KPIs weighted by floor area. Thus, the BIM integration provides a way to configure the sensor automatically and therefore reduce the installation time.

\subsection{IEQ measurement and assessment}

In addition to standard temporal series of data, the Comfort Eye can provide long term indicators and KPI to be used for thermal comfort and IAQ assessment according to EN 16798 methodology that is based on indicators and their boundaries for building's classification [9].

There are four categories (I, II, III, IV) for classification: category $\mathrm{I}$ is the higher level of expectation and may be necessary if the building houses occupants with special requirements (children, elderly, occupants with disabilities, etc.); category IV is the worst. To provide a good level of IEQ, a building is expected to operate at least as category II.

For each IEQ aspect, an hourly or daily indicator $\left(I_{h, i}\right)$ is measured. For every room the number of occupied hours, outside the range $\left(h_{\mathrm{or}, \mathrm{i}}\right)$ is calculated as the number of hours when $I_{h, i} \geq I_{\mathrm{lim}}, I_{\mathrm{lim}}$ is the limit of the indicator determined by the targeted category.

Thus, the percentage of occupied hours outside the range, $\left(\mathrm{POR}_{\mathrm{i}}\right)[\%]$, is calculated as follows (1):

$$
P O R_{i}=\frac{h_{\mathrm{or}, i}}{h_{\mathrm{tot}}} \cdot 100 \% \text {, }
$$

where $h_{\text {tot }}[\mathrm{h}]$ is total occupied hours during the considered period.

The POR of the entire building is finally calculated as the average of the $\mathrm{POR}_{\mathrm{i}}$ for each room, weighted according to the floor area (2):

$$
P O R=\frac{\sum_{i=1}^{n} P O R_{i} \cdot S_{i}}{\sum_{i=1}^{n} S_{i}},
$$

where $n$ is the total number of rooms in the building and $S_{i}$ the floor area of $\mathrm{i}$-th room in the building.

The POR is then used to determine the KPI. A POR of $0 \%$ corresponds to a KPI of $100 \%$, which is the best value achievable. A KPI equal to $100 \%$ means that the indicator is always within the limits of the targeted category. A maximum deviation of $5 \%$ of the POR is considered acceptable. Thus, for POR equal or higher than $5 \%$, the corresponding KPI is $0 \%$ 
(worse case), which means the building is operating for a significant period outside the limits of the targeted category.

To define an assessment scale, a linear interpolation between the minimum POR $(5 \%)$ and best POR $(0 \%)$ is recommended to determine a scale of the KPI between $0 \%$ (worse case) and $100 \%$ (best case).

The analysis is performed only considering the main occupied rooms (e.g., bedrooms, living room, kitchen), and does not consider short-term occupancy and transit areas (e.g., bathrooms, corridors, small storage areas).

Moreover, given the fact that EN 16798 does not cover all the IEQ aspects, such as PM, additional indicators are included.

Following subsections present in detail each indicator used for IEQ assessment. The methodology provides indicators for the calculation of KPIs for the thermal comfort and IAQ assessment.

\subsubsection{Thermal Comfort}

According to ISO 7730 [11] and ISO 7726 [20] "a human being's thermal sensation is mainly related to the thermal balance of his or her body as a whole. This balance is influenced by physical activity and clothing, as well as the environmental parameters: air temperature $\left(T_{\mathrm{a}}\right)$, mean radiant temperature $\left(T_{\mathrm{r}}\right)$, air velocity $\left(V_{\mathrm{a}}\right)$, relative humidity $(\mathrm{RH})$, clothing insulation $\left(I_{\mathrm{cl}}\right)$ and metabolic rate $(M)$.

In a moderate environment, the human thermoregulatory system will automatically attempt to modify skin temperature and sweat secretion to maintain the heat balance. As the definition given in ISO 7730 implies, thermal comfort is a subjective sensation, and it can be expressed with a mathematical model of PMV (3) which is function of four environment parameters and two personal ones:

$$
P M V=\mathrm{f}\left(T_{a}, T_{r}, V_{a}, R H, I_{c l}, M\right) .
$$

The air velocity (in $\mathrm{m} / \mathrm{s}$ ) based on room ventilation system is set to $0.05 \mathrm{~m} / \mathrm{s}$

Metabolic rate $(M)$ is set in function of the usual occupants' activity using a table of typical metabolic rate available in EN7730 [11].

Insulation level $\left(I_{\mathrm{cl}}\right)$ of the clothing generally worn by room occupants. For summer season is set to 0.5 clo and 0.9 clo for the winter season using a table of typical clothing insulations available in EN7730 [11].

The PMV predicts the mean value of votes of a group of occupants on a seven-point thermal sensation scale. As the predicted quality of the indoor thermal environment increases, the $P M V$ value gets closer to 0 (neutral thermal environment). The hourly $P M V$ values need a limit to be set to identify the number of occupied hours outside an acceptable comfort range.

ISO 7730 define the $P M V$ limit for each category, the I category specified as $-0.2<P M V<+0.2$, the II category $0.5<P M V<+0.5$, the III category as $-0.7<P M V<+0.7$ and the IV category $-1<P M V<+1$.

The benchmark is done considering a threshold of a maximum $5 \%$ of operating hours outside the PMV range. The best performance is achieved when there is no deviation outside the design range. So, a linear interpolation between $5 \%$ and $0 \%$ is done.

\subsubsection{Indoor Air Quality}

IAQ is known to have acute and chronic effects on the health of the occupants, generally is expressed in terms of $\mathrm{CO}_{2}$ concentration and ventilation required to contain $\mathrm{CO}_{2}$ levels and
Table 2. PM2.5 Concentration for IEQ classification.

\begin{tabular}{lcc}
\hline Category & 24-hr-PM2.5 $\mathbf{~ g} / \mathrm{m}^{\mathbf{3}}$ & 1-hr PM2.5 $\mathbf{\mu g} / \mathrm{m}^{\mathbf{3}}$ \\
\hline Very good & $0-8.2$ & $0-13.1$ \\
Good & $8.3-16.4$ & $13.2-26.3$ \\
Fair & $16.5-25.0$ & $26.4-39.9$ \\
Poor & $25.1-37.4$ & $40-59.9$ \\
Very poor & 37.5 or greater & 59.9 or greater \\
\hline
\end{tabular}

Table 3. PM 10 Concentration for IEQ classification.

\begin{tabular}{lcc}
\hline Category & 24-hr-PM10 $\boldsymbol{\mu g} / \mathrm{m}^{\mathbf{3}}$ & 1-hr PM10 $\boldsymbol{\mu g} / \mathrm{m}^{\mathbf{3}}$ \\
\hline Very good & $0-16.4$ & $0-26.3$ \\
Good & $16.5-32.9$ & $26.4-52.7$ \\
Fair & $33-49.9$ & $52.8-79.9$ \\
Poor & $50-74.9$ & $80-119.9$ \\
Very poor & 75 or greater & 120 or greater \\
\hline
\end{tabular}

reducing the concentration of indoor air pollutants dangerous for human health [28]. Continuous $\mathrm{CO}_{2}$ monitoring can provide a comprehensive and straightforward way to assess and measure improvements in building ventilation. The IAQ assessment methodology, which provides the $\mathrm{CO}_{2} \mathrm{KPI}$, is defined by EN 16798, and where relevant and needed, IAQ monitoring is enhanced with the monitoring of PM.

The IAQ measurement shall be made where occupants are known to spend most of their time. The KPI aggregates the values at the building level to provide an overall value but to identify critical issues is recommended to analyse all room values.

The hourly $\mathrm{CO}_{2}$ concentration values above outdoor are assessed against a safety threshold to identify the number of hours outside an acceptable comfort range, and the room values are aggregated through a floor-area weighted average.

The $\mathrm{CO}_{2}$ limits for each category, is $550 \mathrm{ppm}$ for the I category, $800 \mathrm{ppm}, 1350 \mathrm{ppm}$ and greater than $1350 \mathrm{ppm}$ for the II, III, and IV category respectively.

According to EN 16789, an acceptable amount of deviation is $5 \%$ of occupied hours. The best performance is achieved when there are no deviations outside the design limit. To define an assessment scale, a linear interpolation between the minimum $(5 \%)$ and best performance $(0 \%)$ is done.

PM is a widespread air pollutant, consisting of a mixture of solid and liquid particles suspended in the air. Commonly used indicators describing PM that are relevant to health refer to the mass concentration of particles with a diameter of less than $10 \mu \mathrm{m}$ (PM10) and particles with a diameter of less than $2.5 \mu \mathrm{m}$ (PM2.5).

The methodology for PM2.5 and PM10 assessment is provided by the Victoria EPA institute (Australia). The categories are obtained according to the hourly and 24-hour rolling average PMs concentration as shown in Table 2 and Table 3 [29].

An acceptable amount of deviation from the optimal category (Very Good) is the $5 \%$. The best performance is achieved when there are no deviations outside the design limit. To define an assessment scale, a linear interpolation between the minimum $(5 \%)$ and best performance $(0 \%)$ is done. 


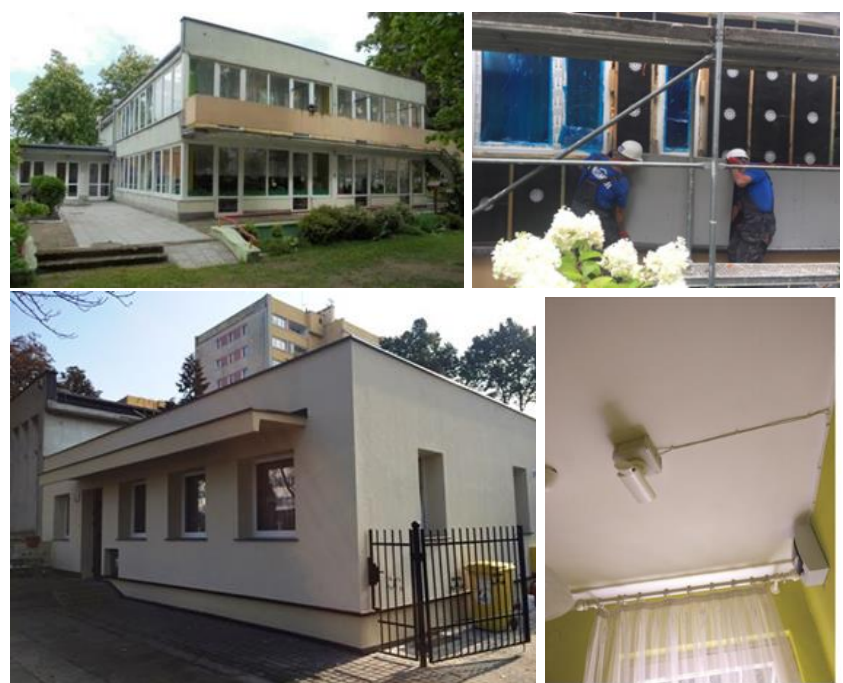

Figure 3. Gdynia Before and After the renovation, Comfort Eye installed in the building.

\section{EXPERIMENTAL APPLICATION}

The Comfort Eye was installed in two rooms of a Nursery of Gdynia and the data collection started in July 2018. The scope of the monitoring was the assessment of the envelope performance and IEQ before and after renovation works (Figure 3).

The demonstration building is a two-story kindergarten building, attended by about 130 children. It was constructed in the year 1965 and it has the function of kindergarten from the beginning. The building volume is $2712 \mathrm{~m}^{3}$ and the built-up area is $464 \mathrm{~m}^{2}$. The main goal of the demonstration was to minimize the energy consumption especially for heating needs through the retrofitting of the envelope (add insulation layer), implementing new windows and improving the aesthetic appearance of the envelope. A comprehensive thermal insulation retrofit should create a continuous insulated envelope around the living accommodation and, ideally, avoid any residual thermal bridging of the structure. The focus of the renovation works was to achieve the targeted quality and performance improving the IEQ.

\section{DISCUSSION OF RESULTS}

Monitoring started in July 2018, the Comfort Eye is still installed and is still acquiring data. The data acquired were analysed for the winter and summer season, before (pre) and after renovation (post), for the assessment of thermal comfort and IAQ building performance and validation of improved IEQ. The renovation works were done from August to October 2019.

The selected periods for the IEQ analysis are August 2018 and August 2020 for the summer season in pre and post renovation state respectively. February 2019 and February 2020 for the winter season in pre and post renovation state respectively. The most significant weeks of the selected periods for the analysis are reported with the relative average outdoor temperature in Table 4.

The weather data were gathered from the Weather the station IGDYNI26 of the Weather Underground web service.

The building is equipped with a centralized heating system with radiators in each room, without a thermostat. No cooling system is present.
Table 4. Selected period for the summer and winter season, pre and post renovation state respectively and outdoor mean temperature.

\begin{tabular}{ccccc}
\hline & \multicolumn{2}{c}{ Summer } & \multicolumn{2}{c}{ Winter } \\
& PRE & POST & PRE & POST \\
\hline \multirow{2}{*}{ Period } & $6 / 8 / 2018$ & $3 / 8 / 2020$ & $25 / 2 / 2019$ & $24 / 2 / 2020$ \\
& $12 / 8 / 2018$ & $9 / 8 / 2020$ & $3 / 3 / 2019$ & $1 / 3 / 2020$ \\
\multirow{2}{*}{ Out T } & $21.3^{\circ} \mathrm{C}$ & $19.0{ }^{\circ} \mathrm{C}$ & $2.9{ }^{\circ} \mathrm{C}$ & $2.8^{\circ} \mathrm{C}$ \\
\hline
\end{tabular}

\subsection{Thermal Comfort}

Concerning thermal comfort, the PMV model was applied using a metabolic rate of 1.2 met (typical office/school activity) and clothing insulation of 0.9 clo, suitable for the end-use of the building and typical climate in Poland in the winter season, and metabolic rate of 1.2 met and clothing insulation of 0.5 clo for the summer season. To compare the pre and post thermographic maps, days with the same average outside temperature have been selected, $6{ }^{\circ} \mathrm{C}(26 / 02 / 2019-20)$ and $25{ }^{\circ} \mathrm{C}(09 / 08 / 2018-20)$ for winter and summer respectively.

The recap of KPIs calculated for the winter and summer season are shown in Table 4 . The mean radiant temperature $\left(T_{\mathrm{r}}\right)$ pre and post renovation is shown in Figure 4.

Figure 5 shows the thermal maps maintaining the same scale in pre and post renovation phases to show the different wall temperatures reached.

Figure $5(a-b)$ shows the thermal maps of the winter season, pre and post renovation, and in Figure $6(\mathrm{a}-\mathrm{b})$ is also possible to observe the trend of the temperature of the wall, of the window and the internal temperature. Although the external temperatures were the same, it can be seen from the graphs and the thermographic map that the temperature of the wall of pre renovation is colder than the wall of post renovation. The monitoring provided evidence that the renovation has increased the insulation capacity of the building.

The building operated for the winter season in Category IV, III, II with a KPI of $0 \%$ before renovation. After renovation operated most of the time in Category I with a KPI of $100 \%$. This turned out to provide an indication of uncomfortable conditions in pre renovation and comfortable conditions in post renovation.

Figure 5 (c-d) show the thermographic maps of the summer season, and in Figure $6(\mathrm{c}-\mathrm{d})$ is also possible to observe the trend of the temperature of the wall, the window, and the internal air temperature. The wall of post renovation is colder than the wall of the pre renovation, this confirms the better insulation of the post renovation. In Figure 5 (c-d) the hottest areas represent the window.

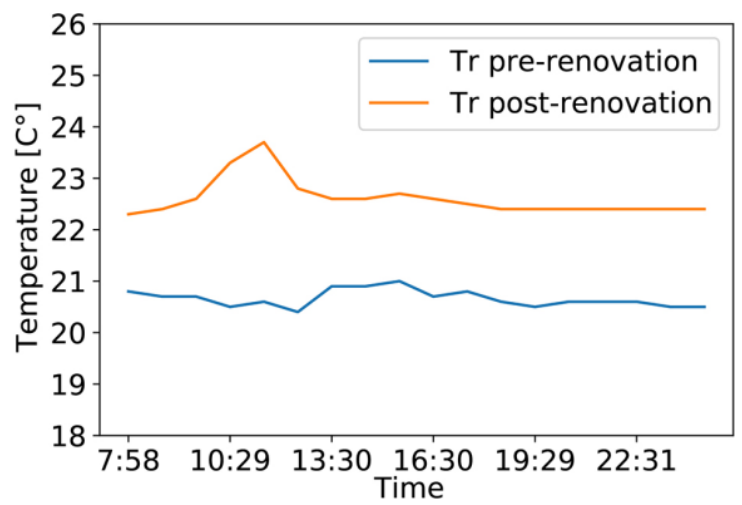

Figure 4. Tr measured with the Comfort Eye before and after renovation. 

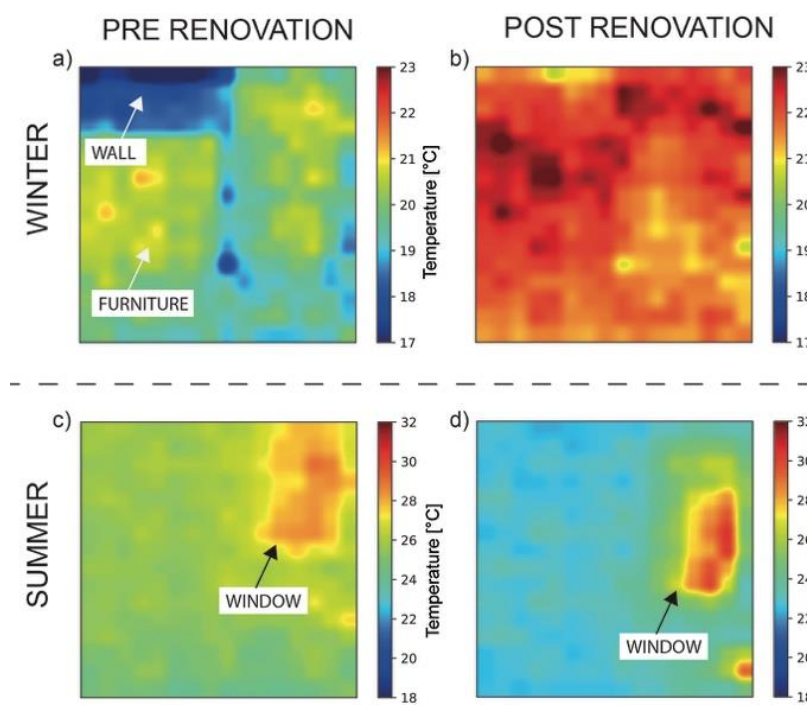

Figure 5. Thermographic Image Pre and Post renovation for the summer and winter season.

For the summer season, the building operated in categories IV, III, II before renovation and in categories III, II, I after renovation. In any case, a KPI of $0 \%$ was registered and this is due to the absence of a cooling system. Thus, only a slight improvement in thermal comfort was registered for the summer season.

The thermal comfort requirements were satisfied for the post renovation. However, in the pre renovation, the detailed analysis on radiant temperatures turned out to provide a clear problem due to the temperatures of the wall exposed to the exterior that, being lower than the other surfaces' temperatures and the air temperature, caused a lower mean radiant temperature (see Figure 4).
Table 5. IEQ KPIs.

\begin{tabular}{crrrr}
\hline \multirow{2}{*}{ KPIs } & \multicolumn{2}{c}{ Summer } & \multicolumn{2}{c}{ Winter } \\
& PRE & \multicolumn{1}{c}{ POST } & \multicolumn{1}{c}{ PRE } & \multicolumn{1}{c}{ POST } \\
\hline Thermal Comfort & $0 \%$ & $100 \%$ & $0 \%$ & $0 \%$ \\
IAQ-CO 2 & $0 \%$ & $20 \%$ & $30 \%$ & $75 \%$ \\
IAQ-PM2.5 & $0 \%$ & $0 \%$ & $85 \%$ & $50 \%$ \\
IAQ-PM10 & $40 \%$ & $60 \%$ & $90 \%$ & $60 \%$ \\
\hline
\end{tabular}

The consequence of lower mean radiant temperature is turned out to provide uncomfortable conditions, resulting in a poor KPI. The post renovation analysis proved that the intervention mitigated that problem thanks to the installation of the envelope panels that increased the wall insulation with a consequent minor drift of the indoor surfaces' temperatures.

\subsection{IAQ}

Table 5 shows the IAQ building performance pre and post renovation.

Considering the winter season, the $\mathrm{CO}_{2} \mathrm{KPI}$ pre renovation is $0 \%$ and improved to $20 \%$ in the post renovation. The KPI of PM2.5 is $0 \%$ for both pre and post renovation. The KPI for the PM10 pre renovation is $40 \%$ and $60 \%$ for the post. No ventilation was installed before and after renovation. The IAQ KPIs registered only a slight improvement that was caused by the introduction of communication with LEDs in the postrenovation phase. The LED light was able to change colour in function of the $\mathrm{CO}_{2}$ and, as shown in Figure 7, it was able to trigger the windows opening by occupants when poor air quality was put in evidence with the light. This underlines how simple communication can improve air quality by changing the habits of occupants. Considering the summer season, the $\mathrm{CO}_{2} \mathrm{KPI}$ is $30 \%$ for the pre renovation and $75 \%$ for post renovation.

\section{PRE RENOVATION}
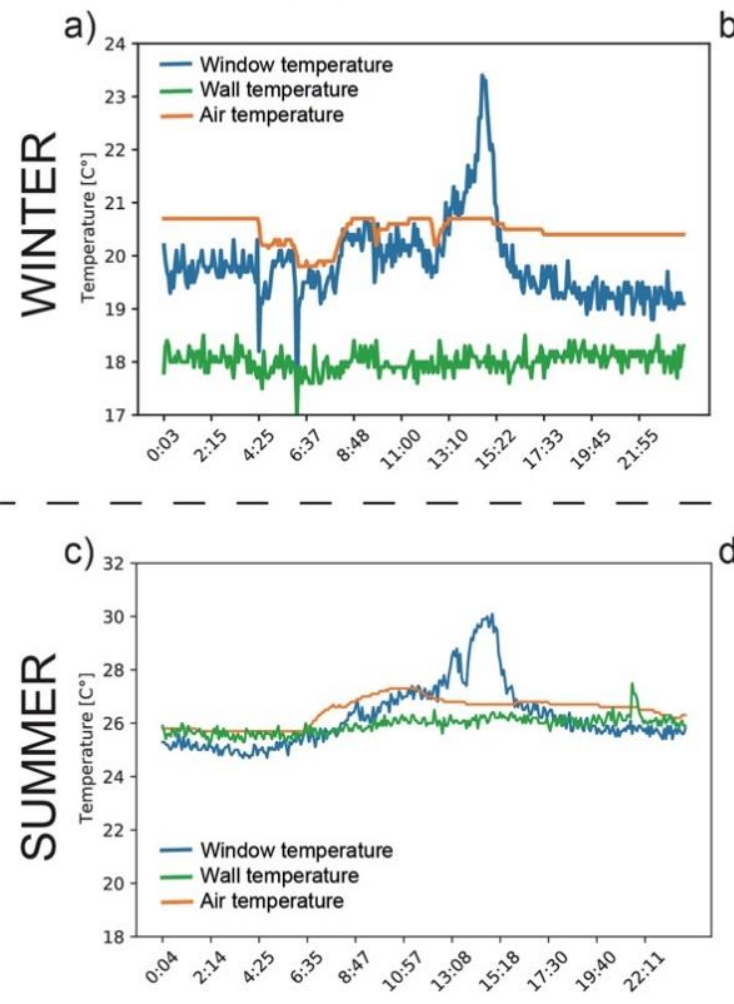

\section{POST RENOVATION}

b)

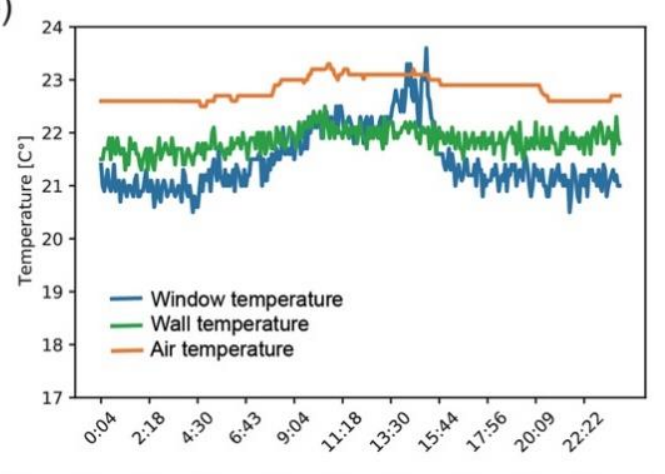

d)

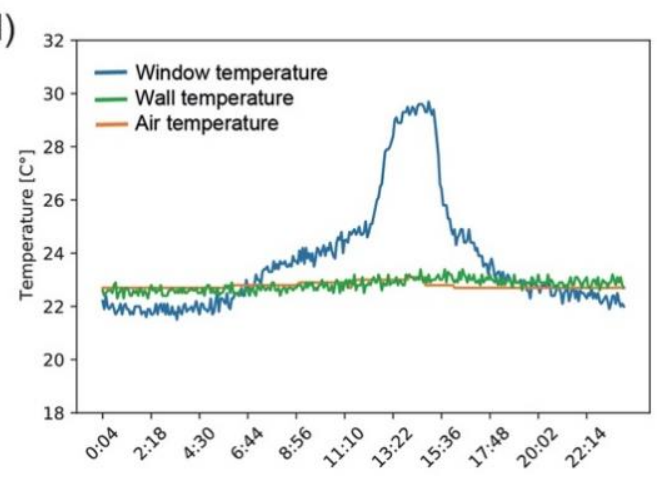

Figure 6. Window, wall and air temperature Pre and Post renovation for the summer and winter season. 


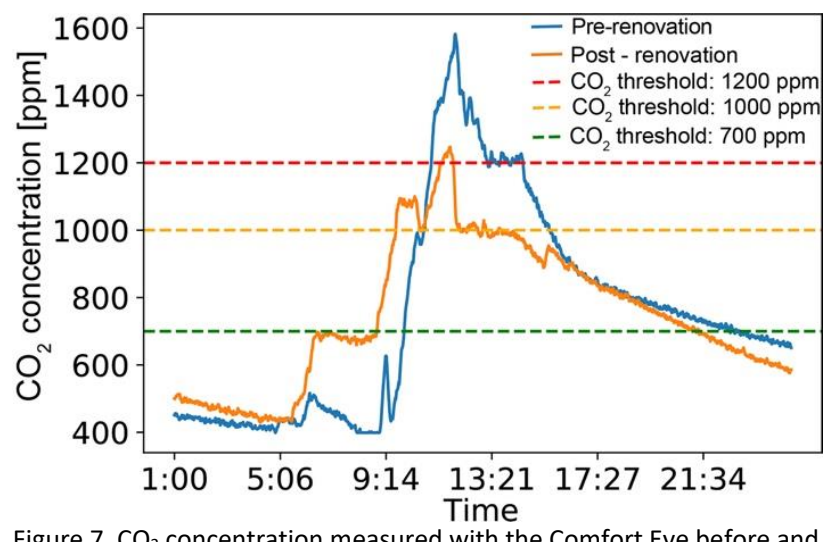

Figure 7. $\mathrm{CO}_{2}$ concentration measured with the Comfort Eye before and after renovation.

The KPI of PM2.5 is $85 \%$ for the pre renovation and $50 \%$ for post renovation.

The KPI for PM10 is $90 \%$ and $60 \%$ for the pre and post respectively. In this case, the final IAQ is provided by natural ventilation because of the opened windows.

Concerning the PMs, a high level of concentrations has been registered. The source of those concentrations requires further investigation. Lower levels of PM2.5 and PM10 were measured in the summer period. The installation of a ventilation system with air purifiers could be considered to mitigate the problem.

The results derived from the case study the feasibility of the proposed IoT innovative system for continuous measurement of all parameters required for an accurate analysis of the IEQ. This system allows to carry out a long-term monitoring and, therefore, to evaluate of the IEQ in the most significant seasons.

In the presented application, both communication means were used. The occupants were able to take advantage of the realtime IAQ advice by LEDs. The web-app was used to generate seasonal reports with IEQ KPIs that were used to evaluate the optimal renovation strategy and the impact of the renovation works.

\section{CONCLUSION}

The Comfort Eye is an innovative and non-intrusive solution, allowing the implementation of an IoT sensing system for a complete, continuous, and long-term monitoring of the IEQ. Together with the sensing device, a set of performance indicators has been developed to assess the overall quality in terms of thermal comfort and indoor air quality.

This paper demonstrates the applicability and advantages of the proposed measurement device with the application to a real case study. A pre and post renovation analysis was performed to validate the developed monitoring methodology.

The ceiling node, given the high spatial and temporal resolution compared with traditional tools, provides two main advantages. First, multipoint measurements of the mean radiant temperature with only one sensor. Given the geometry of the room, several locations can be chosen to apply the calculation of the mean radiant temperature (e.g., near and far from the window). Second, together with information about comfort, thermal maps of the indoor surfaces are available and can be used to track the thermal performance of the building envelope (e.g., tracking surface temperature of the external wall, recognizing cool zones etc.).

The IR scanner of the Comfort Eye turned out be useful to accurately detect the thermal comfort issues because of its capability of measuring thermal maps and mean radiant temperatures. To this aim, the accuracy and completeness of BIM data, such as material emissivity and geometry, are pivotal and should be guaranteed by a standardized BIM modeling approach.

The monitoring allowed to identify the causes of the thermal discomfort. In the pre-renovation phase the building operated for a significant period (more than $5 \%$ ) out of the targeted category with a KPI of $0 \%$ (worst conditions). The average radiant temperature is the most significant cause of discomfort, the building had poor thermal insulation of the walls. The monitoring has confirmed that by working on the causes, the performance of the building can be improved with a consequent minor drift of the indoor surfaces' temperatures. In the post renovation phase the building operates within the limits of the targeted category with a KPI of $100 \%$.

The desk node allows a more detailed view of the performance of the building, providing information regarding the air quality. Furthermore, the large set of information available from the measured data can be used to identify IEQ problems and their origin. Such advanced knowledge of the building performance allows a better design of the renovation.

Concerning the IAQ, the monitoring demonstrated that the building operates in the pre and post renovation phases outside the limit of the targeted category (more than $5 \%$ ). No mechanical ventilation system was installed with the renovation and the installation of a ventilation system with air purifiers could be considered to mitigate the problem. The IAQ KPIs registered only a slight improvement that was caused by the introduction of communication with LEDs in the post- renovation phase.

The capability of communicating the status of indoor air quality with the simple LED colour based on real time measurements, triggered occupants' actions with the scope of restoring the required environmental quality.

Future developments will provide improvements of the measurement system to enhance the Plug\&Play installation and to include additional sensors for the evaluation of other IEQ aspects, as visual and acoustic comfort.

\section{ACKNOWLEDGEMENT}

This research has received funding from the P2Endure.

The P2Endure research project (https://www.p2endureproject.eu/en) is co-financed by the European Union within the H2020 framework programme with contract no. 723391. The authors want to thank the project partners for the useful discussions and collaboration.

\section{REFERENCES}

[1] EU Building Stock Observatory. Online [Accessed 17 December 2021]

https://ec.europa.eu/energy/topics/energy-efficiency/energyefficient-buildings/eu-bso en

[2] A. G. Kwok, N. B. Rajkovich, Addressing climate change in comfort standards, 2010, Building and Environment, vol. 45, issue 1, pp. 18-22.

[3] A. H. Yousef, M. Arif., M. Katafygiotou, A. Mazroei, A. Kaushik, E. Elsarrag, Impact of indoor environmental quality on occupant well-being and comfort: A review of the literature. International Journal of Sustainable Built Environment, 2016, vol. 5, pp. 1-11. DOI: $10.1016 /$ i.buildenv.2009.02.005

[4] I. Mujan, A. S. Anđelković, V. Munćan, M. Kljajić, D. Ružić, Influence of indoor environmental quality on human health and productivity - A review, Journal of Cleaner Production, 2019, vol. 
217, pp. 646-657.

DOI: $10.1016 /$ i.jclepro.2019.01.307

[5] E. Oldham, H. Kim,IEQ Field Investigation in HighPerformance, Urban Elementary Schools. Atmosphere 2020, 11, 81. DOI: $10.3390 /$ atmos11010081

[6] K. Azuma, N. Kagi, H. Kim, M. Hayashi, Impact of climate and ambient air pollution on the epidemic growth during COVID-19 outbreak in Japan. Environ Res. 2020, 190, 110042.

DOI: $10.1016 /$ i.envres.2020.110042

[7] M. A. Zoran, R. S. Savastru, D. M. Savastru, M. N. Tautan, Assessing the relationship between surface levels of PM2.5 and PM10 particulate matter impact on COVID-19 in Milan, Italy, Science of The Total Environment, Volume 738, 2020, 139825. DOI: $10.1016 /$ j. scitotenv.2020.139825

[8] A. M. Atzeri, F. Cappelletti, A. Tzempelikos, A. Gasparella, Comfort metrics for an integrated evaluation of buildings performance,Energy and Buildings, Volume 127, 2016, pp. 411424.

DOI: $10.1016 /$ i.enbuild.2016.06.007

[9] CEN, EN 16798 Indoor environmental input parameters for design and assessment of energy performance of buildingsaddressing indoor air quality, thermal environment, lighting and acoustics. CEN, 2019].

[10] A. Kylili, P. A. Fokaides, P. A. L. Jimenez, Key Performance Indicators (KPIs) approach in buildings renovation for the sustainability of the built environment: A review, Renewable and Sustainable Energy Reviews, vol. 56, 2016, pp. 906-915. DOI: $10.1016 /$ i.rser.2015.11.096

[11] ISO, ISO 7730 Ergonomics of the thermal environment Analytical determination and interpretation of thermal comfort using calculation of the PMV and PPD indices and local thermal comfort criteria, International Standardization Organization, Geneva (2005)

[12] D. Khovalyg, O. B. Kazanci, H. Halvorsen, I. Gundlach, W. P. Bahnfleth, J. Toftum, B. W. Olesen, Critical review of standards for indoor thermal environment and air quality, Energy and Buildings, vol. 213, 2020.

DOI: $10.1016 /$ i.enbuild.2020.109819

[13] Y. Song, F. Mao and Q. Liu, Human Comfort in Indoor Environment: A Review on Assessment Criteria, Data Collection and Data Analysis Methods, in IEEE Access, vol. 7, 2019, pp. 119774-119786. DOI: $\underline{10.1109 / A C C E S S .2019 .2937320}$

[14] L. Claudi, M. Arnesano, P. Chiariotti, G. Battista, G. M. Revel, A soft-sensing approach for the evaluation of the acoustic comfort due to building envelope protection against external noise, Measurement, vol. 146, 2019, pp. 675-688. DOI: $10.1016 /$ i.measurement.2019.07.003

[15] T. S. Larsen, L. Rohde, K. T. Jønsson, B. Rasmussen, R. L. Jensen, H. N. Knudsen, T. Witterseh, G. Bekö, IEQ-Compass - A tool for holistic evaluation of potential indoor environmental quality, Building and Environment, vol. 172, 2020, 106707. DOI: $10.1016 /$ j.buildenv.2020.106707
[16] B. D. Hunn, J. S. Haberl, H. Davie, B. Owens, Measuring commercial building performance protocols for energy, water, and indoor environmental quality (2012), ASHRAE Journal, 54 (7), pp. 48-59.

[17] F. Seri, M. Arnesano, M.M. Keane,G.M. Revel,Temperature Sensing Optimization for Home Thermostat Retrofit. Sensors 2021, 21, 3685 DOI: $10.3390 / \mathrm{s} 21113685$

[18] I. Atmaca, O. Kaynakli, A. Yigit, Effects of radiant temperature on thermal comfort, Building and Environment, vol. 42, issue 9, 2007, Pages 3210-3220. DOI: $10.3390 /$ BUILDINGS11080336

[19] G. Gan, Analysis of mean radiant temperature and thermal comfort. Building Services Engineering Research and Technology 2001, 22(2), pp.95-101. DOI: $10.1191 / 014362401701524154$

[20] ISO, ISO 7726. 2002. Ergonomics of the thermal environment instruments for measuring physical quantities". International Standardization Organization, Geneva, 2002.

[21] G. M. Revel, E. Sabbatini, M. Arnesano, Development and experimental evaluation of a thermography measurement system for real-time monitoring of comfort and heat rate exchange in the built environment, Measurement Science and Technology, 2012, 23(3). DOI: $10.1088 / 0957-0233 / 23 / 3 / 035005$

[22] G. M. Revel, M. Arnesano, F. Pietroni, Development and validation of a low-cost infrared measurement system for realtime monitoring of indoor thermal comfort, Measurement Science and Technology, vol. 25(085101), 2014L. DOI: $10.1088 / 0957-0233 / 25 / 8 / 085101$

[23] L. Zampetti, M. Arnesano, G. M. Revel, Experimental testing of a system for the energy-efficient sub-zonal heating management in indoor environments based on PMV, Energy and Buildings, vol. 166, 2018, pp. 229-238.

DOI: $10.1016 /$ i.enbuild.2018.02.019

[24] X. P. Maldague, Theory and Practice of Infrared Technology for Nondestructive Testing. Wiley-Interscience, 2001, ISBN: 978-0471-18190-3.

[25] Health Canada, Residential indoor air quality guidelines: Carbon dioxide, 2021.

[26] REHVA - Federation of European Heating, Ventilation and Air Conditioning Associations, $\mathrm{CO}_{2}$ monitoring and indoor air quality. Online [accessed 17 December 2021] https://www.rehva.eu/rehva-journal/chapter/co2-monitoringand-indoor-air-quality

[27] IfcOpenShell Academy. Online [Accessed 17 December 2021] https://academy.ifcopenshell.org/

[28] P. Wargocki, D. P. Wyon, J. Sundell, G. Clausen, P. O. Fanger, The effects of outdoor air supply rate in an office on perceived air quality, sick building syndrome (SBS) symptoms and productivity, Indoor Air 10 (2000) 222-236. DOI: $10.1034 / \mathrm{j} .1600-0668.2000 .010004222 . \mathrm{x}$

[29] EPA Victoria, Air pollution in Victoria - a summary of the state of knowledge, Publication 1709 August 2018. 